\title{
THE SELF-ORGANISING FEATURE MAP IN THE DETECTION OF EPILEPTIFORM TRANSIENTS IN THE EEG
}

\author{
Christopher J. James ${ }^{1^{*}}$, Student Member, IEEE, Richard D. Jones ${ }^{2}$, Senior Member, IEEE, \\ Philip J. Bones ${ }^{1}$, Senior Member, IEEE, and Grant J. Carroll ${ }^{3}$ \\ ${ }^{1}$ Department of Electrical and Electronic Engineering, University of Canterbury \\ ${ }^{2}$ Department of Medical Physics \& Bioengineering, Christchurch Hospital \\ ${ }^{3}$ Department of Neurology, Christchurch Hospital \\ Christchurch, New Zealand
}

\begin{abstract}
A system is proposed which utilises Kohonen's selforganising feature map (SOFM) to help detect the presence of epileptiform transients in the EEG. The system consists of a feature extractor, which employs a mimetic approach to detect candidate epileptiform transients (CETs) on individual channels in the multichannel recording, followed by a trained SOFM.

The SOFM system is proposed as a single channel module of a larger system to detect multichannel epileptiform events by incorporating the outputs of sixteen such modules.

The SOFM was trained with CETs obtained from 16-channel bipolar EEG segments of nine patients and fine-tuned through Kohonen's learning vector quantisation technique (LVQ2). Measures of the sensitivity and specificity of the trained map were obtained by presenting a subset of CETs to the SOFM which had been graded by two to three electroenceph. alographers as being true or false. The results obtained show that the trained SOFM has a sensitivity of $63 \%$ and a specificity of $79 \%$ for a $16 \times 16$ SOFM.
\end{abstract}

\section{INTRODUCTION}

$\mathrm{T}$ HE detection of epileptiform transients (ETs) in the electroencephalogram (EEG) is of particular importance in the diagnosis of epilepsy. Many methods for the automated detection of epileptiform transients have been developed but, for the most part, with limited success $[1,2]$. With the introduction of artificial neural networks (ANNs) several authors have performed pattern recognition on the EEG by training ANNs on large numbers of known ETs, e.g. [3]. This, however, has also a limited performance as well as the disadvantage of requiring a large number of ETs, all of which need to be graded by expert electroencephalographers (EEGers).

The method described here makes extensive use of unsupervised learning methods to form a realistic representation of the problem by using large numbers of unlabelled inputs followed by classification using a small quantity of labelled inputs. Kohonen's self-organising feature map (SOFM) [4] was chosen for this purpose.

\section{METHODS}

\section{A. Subjects}

Sixteen channels of EEG where recorded simultaneously from scalp electrodes both for referential and bipolar montages. The amplified EEG was bandpass filtered between 0.5 and $70 \mathrm{~Hz}$, sampled at $200 \mathrm{~Hz}$, digitised to 12 bits, and stored for later off-line processing. Recordings from nine patients with epileptiform activity were obtained, totalling 205 min of 16-channel bipolar EEG. These were then split into two equal sets: a training set and a test set.

\section{B. Ist stage: Mimetic stage}

In order to reduce the amount of data being put forward to the ANN, a mimetic stage was introduced (Fig. 1). This stage worked by scanning the EEG on all channels and looking for vertices. A number of parameters were calculated for each vertex, including: pre-vertex slope, post-vertex slope, sharpness, relative amplitude and duration. Each parameter was then thresholded and a pass/fail indicator set for each waveform. The thresholds were set to give a high sensitivity for true ETs. Once a waveform had passed the thresholds, all other waveforms on the same bipolar chain werc extracted, provided they were within $50 \mathrm{~ms}$ of the primary vertex - these constituted the candidate epileptiform transients (CETs). With the CET locations determined, a segment of the raw EEG was extracted about each vertex for a width of 41 samples $(205 \mathrm{~ms})$. In addition, a number of contextual parameters were calculated from $1 \mathrm{~s}$ of background EEG around each CET (average duration, amplitude, and sharpness) and stored along with the raw EEG segment and a pass/fail-threshold indicator.

The CETs were then grouped into events around a given bipolar chain and the events were then labelled by two (in some cases three) EEGers as true or false, the final label being based on a consensus amongst the EEGers.

\footnotetext{
* c-mail: jamescj@elec.canterbury,ac.nz
} 


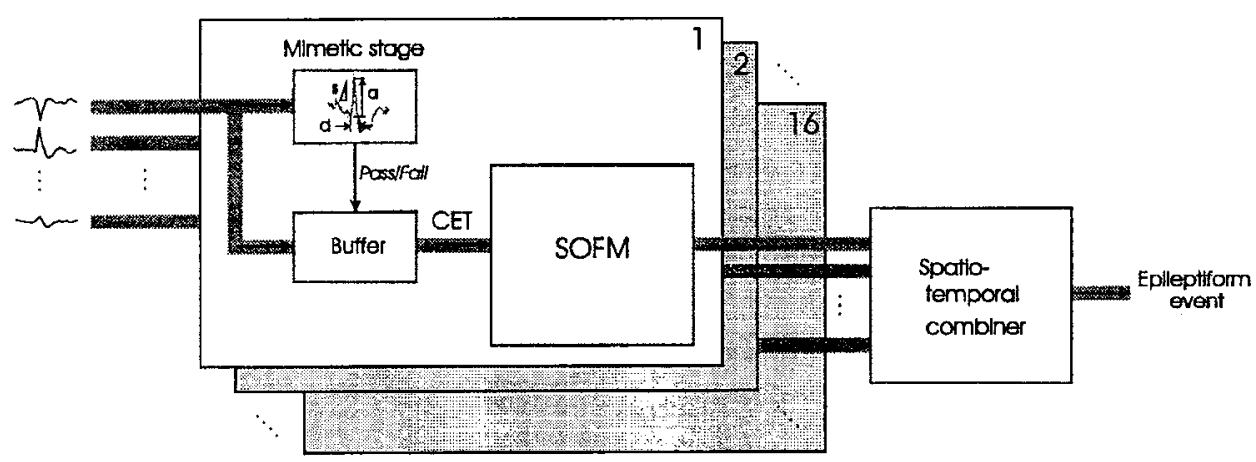

Fig. 1. A diagram depicting the SOFM module and its part in the proposed multichannel ET detector.

\section{2nd stage: Self-organising feature map}

The SOFM is a lattice type ANN whose neurons become specifically tuned to various input signals through an unsupervised learning process. It does not require prior information about the statistics of the inputs. We adopted a square two-dimensional SOFM of varying order $N$.

The raw EEG segment forming a CET, along with its corresponding contextual information and the pass/failthresholds flag, were treated as a 45 dimensional feature vector which was presented to the SOFM (Fig. 1).

As there was a large imbalance in numbers between true and false CETs, false CETs were randomly removed from the training set until the numbers became balanced.

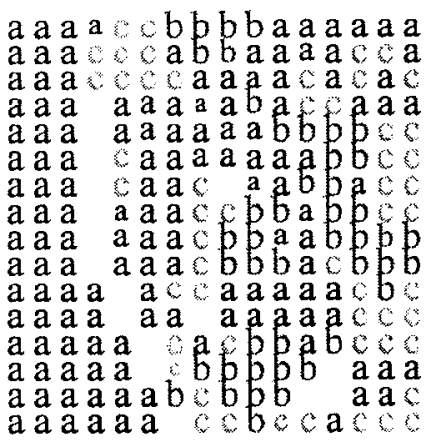

Fig, 2. The labels assigned to each of the neurons in the $16 \times 16$ SOFM: a:false ET, b:true ET (+ve going) \& c:true ET (-ve going). The size of the label represents the confidence level with which each class is represented.

\section{Performance}

The training set was presented 16 times to the SOFM (in random order). Once the training was complete, a subset of the training set (the calibration set), was presented to the trained SOFM and each neuron assigned a class label according to its best matching response (Fig. 2). The SOFM's weights were subsequently 'fine-tuned' by the learning vector quantisation (LVQ) technique proposed by Kohonen [4] (LVQ2 was implemented). The fine-tuned map was then presented with the test-set CETs and measures of the sensitivity and selectivity obtained.
The map size $N$ was varied and the whole training/testing procedure was repeated while observing the performance of the map and the mean Euclidean distance of the map's weights at successive iterations during training.

\section{RESULTS}

The optimum map size was found to be $16 \times 16$. Above $N=16$, increases in performance, as well as decreases in the mean Euclidean distance between the weights of the map, were negligible. This yielded a sensitivity of $63 \%$ and specificity of $79 \%$ to true CETs.

\section{DISCUSSION}

Preliminary results indicate that the SOFM followed by LVQ2 is well suited to detecting ETs in the single channel EEG. It is also important to note that the sensitivity of the full 16-channel SOFM system will, in fact, be considerably greater for multichannel events than a single SOFM (as indicated here) is for single-channel CETs. A multichannel performance analysis is well in progress.

We consider that this new single-channel approach will play an important part in a high performance ET detection system. This larger system will utilise spatial and temporal contextual information (considered essential for high accuracy ET detection [2]) by, in part, incorporating and integrating the outputs of 16 identical SOFM stages (Fig. 1).

\section{REFERENCES}

[1] J. Gotman, "Automatic recognition of interictal spikes", in Long term monitoring in epilepsy EEG supplement No. 37, J. Gotman, J.R. Ives, and P. Gloor, Eds., pp. 99-114. Elsevier, 1985.

[2] A.A. Dingle, R.D. Jones, G.J. Carroll and W.R. Fright, "A multistage system to detect epileptiform activity in the EEG", IEEE Trans. Biomed. Eng., vol. 40, pp. 1260-1268, 1993.

[3] W.R.S. Webber, B. Litt, K. Wilson and R.P. Lesser, "Practical detection of epileptiform discharges (EDs) in the EEG using an artificial neural network: a comparison of raw and parameterized EEG data", Electroenceph. Clin. Neurophysiol., vol. 91, pp. 194-204, 1994

[4] T. Kohonen, "The self-organizing map", Proc. IEEE, vol. 78, pp. 1464-1480, 1990. 